\title{
CONNAISSANCE DU FOYER AUVERgNAT D'ÉCHINOCOCCOSE ALVÉOLAIRE
}

\section{Recherche de l'hôte intermédiaire, description des lésions.}

\author{
A. F. PÉTAVY* et S. DEBLOCK**
}

RÉSUMÉ. Vingt trois campagnols terrestres adultes (Arvicola terrestris scherman Shaw Mammifère microtidé) sont porteurs d'au moins une larve d'Echinococcus multilocularis parasite du foie, sur 943 rongeurs piégés en un an d'août 1981 à août 1982, dans sept communes différentes d'Auvergne (départements du Puy-de-Dòme et du Cantal), soit 2,44\%. Selon les gîtes, les pourcentages moyens d'animaux parasités varient de 0 à 4,65\% ; selon le mois de l'année, ces pourcentages varient de 0,6 à 3,6 dans les gîtes actifs, des pointes exceptionnelles s'avérant possibles $117,5 \%$ en janvier dans le gîte d'Allanche, à prévalence annuelle de $3 \%$ ). Les foyers favorables restent actifs toute l'année.

Les divers aspects des lésions anatomo-pathologiques des foies sont décrits, en particulier ceux consécutifs à la présence de larves jeunes. $60 \%$ des larves en effet — soit $14 / 23$ — sont des hydatides jeunes, de petite taille, et non fertiles. Les diverses étapes de la maturation sont répertoriées et classées d'après les tailles et les configurations histologiques observées. $8,7 \%$ des larves sont fertiles (soit 2/23). L'une de ces dernières paraît en voie de dégénérescence, formée de grandes vessies gigognes nombreuses, profuses en membranes cuticulaires et pauvres en vésicules proligères dont les rares protoscolex sont vivants ou dégénérés. La seconde simule une larve pluriloculaire d'E. granulosus bien circonscrite par l'adventice; elle est riche de milliers de protoscolex matures en excellent état physiologique. Ces observations démontrent la bonne réceptivité de l'hôte intermédiaire mis en évidence en Auvergne à la souche locale du parasite, mais semble attester sa mauvaise aptitude relative à conduire le développement de la larve jusqu'à son terme.

La dispersion de l'enzootie échinococcique alvéolaire chez les animaux sauvages en Auvergne, tant chez les renards (parasités au taux de 8,5\% )que chez les rongeurs, constitue une menace permanente de contamination de la population rurale ou des vacanciers fréquentant le foyer; le risque paraît faible ( 5 cas humains diagnostiqués en 10 ans) mais non nul.

\section{Investigations on the Auvergnan focus of alveolar echinococcosis in France. Research about the intermediate host. Parasite-host relationships.}

SUMMARY. Echinococcus multilocularis larvae occured in $23(2.44 \%)$ of the 943 adult vole rats (Arvicola terrestris scherman Shaw, Mammal Microtidae) collected in the montagnous center of France (Massif Central, départements of Puy-de-Dòme and Cantal, province of Auvergne) and

\footnotetext{
* Laboratoire de Parasitologie, Facultés de Médecine et de Pharmacie, 8, avenue Rockefeller, F 69373 Lyon Cedex. Lille cedex.

** Laboratoire de Parasitologie, U.E.R. de Pharmacie de Lille, rue du Professeur-Laguesse, F 59045 Accepté le 21 janvier 1983.
} 
obtained from August 1981 to Aug. 1982 in seven different localities. According to this localities, the average percentage of parasited voles changes from zero to $4.65 \%$; according to the month of the year, these percentage change from 0.6 to 3.6 in the active sites while unusual picks can be reached (17.5 in January in the Allanche focus with yearly prevalence of $3 \%$ ). The favorable focuses remain active the whole year. The different aspects of the anatomo-pathological changes of the livers are described, particularly those due to young larvae. $14(60 \%)$ of the 23 larvae are young, small and unfertile hydatids. Only $2(8.7 \%)$ of the 23 are fertile. One of these two larvae seems to degenerate and produces a few protoscolex alive ; the other simulates a plurilocular larva of $E$. granulosus, restricted to a single lobe well delimitated by the fibrous adventicia and produces the protoscolex in great number. These observations prove the good receptivity of the vole rat to the local strain of the parasite but seem to attest its relative inaptitude to bring the larva to maturity. The dispersion of the alveolar echinococcosis among the wild animals in Auvergne ( 8.5 of the foxes parasited and $2.44 \%$ of the vole rats) realised a permanent threat of contamination as regard rural population or people on holiday during the summer. The risk seems to be week ( 5 human cases of hepatic alveolar hydatid disease for ten years) bur actual.

\section{I - Introduction}

Les foyers d'échinococcose alvéolaire se situent classiquement en France à l'est du pays, de Charleville à Chambéry, dans les régions collinaires ou montagneuses de Lorraine, de Franche-Comté et de Savoie. Rey et coll., 1977 et 1978 puis Bourée et coll., 1978 attirent les premiers l'attention sur trois cas chirurgicaux d'échinococcose alvéolaire humaine déclarés en Auvergne. Depuis ces deux dates, deux cas supplémentaires sont encore diagnostiqués dans la même région (tableau I).

TABleau I. - Mise en évidence du foyer d'échinococcose multiloculaire dans le Massif Central. Cas humains.

\begin{tabular}{|c|c|c|c|c|c|}
\hline $\begin{array}{c}\text { Année de } \\
\text { publication }\end{array}$ & No & Sexe & $\begin{array}{l}\text { Age et } \\
\text { profession }\end{array}$ & Localité & $\begin{array}{l}\text { Année du } \\
\text { diagnostic }\end{array}$ \\
\hline \multirow{3}{*}{1977} & 1 & $\widehat{\sigma}$ & \multirow{3}{*}{$\begin{array}{l}38 \text { ans } \\
\text { agriculteur } \\
53 \text { ans } \\
\text { commerçante }\end{array}$} & \multirow{3}{*}{$\begin{array}{l}\text { Égliseneuve } \\
\text { d'Entraigues } \\
\text { Riom-ès- } \\
\text { Montagne }\end{array}$} & \multirow{2}{*}{1970} \\
\hline & 2 & 우 & & & \\
\hline & & & & & 1975 \\
\hline \multirow{2}{*}{1978} & 3 & 우 & $\begin{array}{l}52 \text { ans } \\
\text { cultivatrice }\end{array}$ & Allanche & 1976 \\
\hline & 4 & $\hat{\sigma}$ & 45 ans & "séjours en Creuse» & 1976 \\
\hline \multirow{2}{*}{$\begin{array}{l}\text { Cas non- } \\
\text { publiés }\end{array}$} & 5 & $\hat{\sigma}$ & \multirow{2}{*}{$\begin{array}{l}58 \text { ans } \\
\text { cultivateur } \\
68 \text { ans } \\
\text { cultivateur }\end{array}$} & \multirow{2}{*}{$\begin{array}{l}\text { Égliseneuve } \\
\text { d'Entraigues } \\
\text { Riom-ès- } \\
\text { Montagne }\end{array}$} & \multirow{2}{*}{1980} \\
\hline & 6 & $\hat{0}$ & & & \\
\hline
\end{tabular}

Pétavy et coll., 1980 démontrent que la parasitose humaine tire son origine d'un foyer naturel actif dans le Massif Central, dont le renard constitue l'un des maillons du cycle épidémiologique : 7,2\% des renards communs (Vulpes vulpes $L$.) hébergent 
la forme adulte du cestode Echinococcus multilocularis Leuckart, 1853 lors de 69 autopsies (dont 61 pratiquées en Auvergne) ${ }^{1}$. Aux 5 renards parasités de 1980, s'en ajoute un supplémentaire autopsié en 1982, ce qui élève le pourcentage d'animaux parasités à $8,57 \%$ pour la série des 70 renards (Puy-de-Dôme. : 6,81\% et Cantal : 11,1\%). (Tableau II et carte 1.)

TABleau II. - Mise en évidence de l'hôte définitif naturel d'Echinococcus multilocularis dans le Massif Central.

\begin{tabular}{|c|c|c|c|c|c|}
\hline $\begin{array}{c}\text { Dates } \\
\text { d'autopsies }\end{array}$ & Départements & $\begin{array}{c}\text { Nombre } \\
\text { d'autopsies }\end{array}$ & $\begin{array}{l}\text { Renards } \\
\text { positifs }\end{array}$ & $\begin{array}{c}\% \text { par } \\
\text { département }\end{array}$ & $\begin{array}{l}\text { Localisation } \\
\text { géographique }\end{array}$ \\
\hline 1977 & Allier & 6 & 0 & & \\
\hline Avril, mai & Puy-de-Dôme & 43 & 2 & $4,65 \%$ & $\begin{array}{l}\text { Mozac, } \\
\text { Entraigues }\end{array}$ \\
\hline $\begin{array}{l}\text { Décembre } \\
\text { Sept., déc. }\end{array}$ & $\begin{array}{l}\text { Creuse } \\
\text { Cantal }\end{array}$ & $\begin{array}{r}2 \\
18\end{array}$ & $\begin{array}{l}1 \\
8\end{array}$ & $11,1 \%$ & $\begin{array}{l}\text { Flayat } \\
\text { Riom-ès-Mont. } \\
\text { Oradour }\end{array}$ \\
\hline Juill. 1982 & Puy-de-Dôme & 1 & 1 & & $\begin{array}{l}\text { Égliseneuve } \\
\text { d'Entraigues }\end{array}$ \\
\hline
\end{tabular}

Houin et coll., 1980 mettent en évidence le rôle d'hôte intermédiaire naturel du cestode joué en Franche-Comté, par le campagnol terrestre Arvicola terrestris scherman Shaw. Ce rongeur microtidé est présent en Auvergne dans des biotopes analogues à ceux du Jura. Constitue-t-il pareillement dans le Massif Central le deuxième maillon de la chaîne épidémiologique de la parasitose ? La capture et l'autopsie de 943 individus de l'espèce au cours des années 1981 et 1982 apportent des éléments de réponse à la question mais sans résoudre, semble-t-il, le problème de façon satisfaisante et définitive.

\section{II - Matériels et méthodes}

Les lieux de piégeage des grands campagnols résultent d'un choix assis sur un faisceau de présomptions a priori favorables réunissant l'existence locale de cas humains d'échinococcose alvéolaire, de renards parasités par E. multilocularis adultes et d'une densité suffisante d'Arvicola.

Les rongeurs sont capturés en sous-sol à la boite Scherman, complétée selon chaque opportunité par la pose simultanée de un ou deux pièges à pince. Une anesthésie à l'éther tue les animaux capturés vivants. Toutes les dépouilles d'un même piégeage sont conservées au frais en sacs plastiques numérotés, jusqu'à leur examen sommaire pratiqué dans un délai maximal de 24 à 48 heures sur le terrain même ou au laboratoire. Poids et sexes sont notés. A l'autopsie, le foie est réséqué et examiné

I. L'Auvergne s'étend sur les départements du Puy-de-Dôme et du Cantal. 
en priorité. Toute lésion macroscopiquement décelable est prélevée pour être fixée dans le liquide de Baker (formol-calcium) en vue de l'examen ultérieur anatomopathologique détaillé. Lors de ce dernier, les lésions suspectées d'être d'origine parasitaire sont d'abord coupées en deux parts, de préférence en lisière, et une fine tranche de tissu ( $1 / 2$ mm environ) est détachée à main levée au rasoir et déposée sur lame dans une goutte de gomme-chloral, aux fins préalable d'examen rapide d'orientation. 1) En présence d'une cysticercose, le scolex est prélevé et examiné à part. Les crochets sont mesurés à plat après écrasement ménagé du scolex ou du rostre, sous lamelle et dans la gomme-chloral, qui fournit une préparation permanente après dessication. 2) En absence de cysticercose, l'examen histologique est conduit selon les données classiques de routine (coupes à la paraffine à $10 \mu \mathrm{m}$, coloration au Wheatley ou à l'hémalun-éosine, montage au baume). Ce dernier examen précise la nature réelle des lésions ayant justifié le prélèvement.

Origines géographiques, dates et nombre de capture des campagnols.

Les piégeages des Arvicola ont commencé en août 1981 et se sont poursuivis jusqu'en août 1982. Communes, altitudes, et dates de captures des 943 animaux sont précisées dans le tableau III et la carte $n^{\circ} 2$. Ces animaux sont constitués d'adultes des deux sexes du poids moyen d'une centaine de grammes pour la très grande majorité d'entre eux.

TABLEAU III. - Origines, dates et nombres des captures d'Arvicola

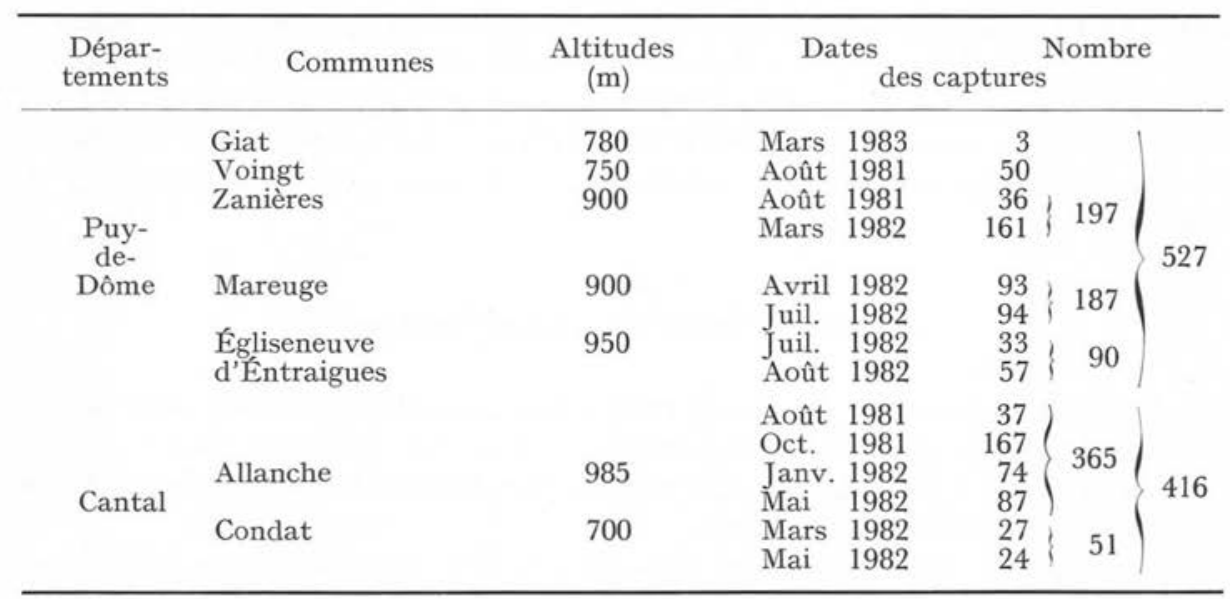

\section{III — Résultats des observations}

8,58 \% des Arvicola piégés (81/943) hébergent des larves hépatiques de cestode d'espèces diverses, dont 23 sont déterminées en tant qu'hydatides alvéolaires. Ces 23 cas représentant $2,44 \%$ des prises, se répartissent au sein des lieux de capture 


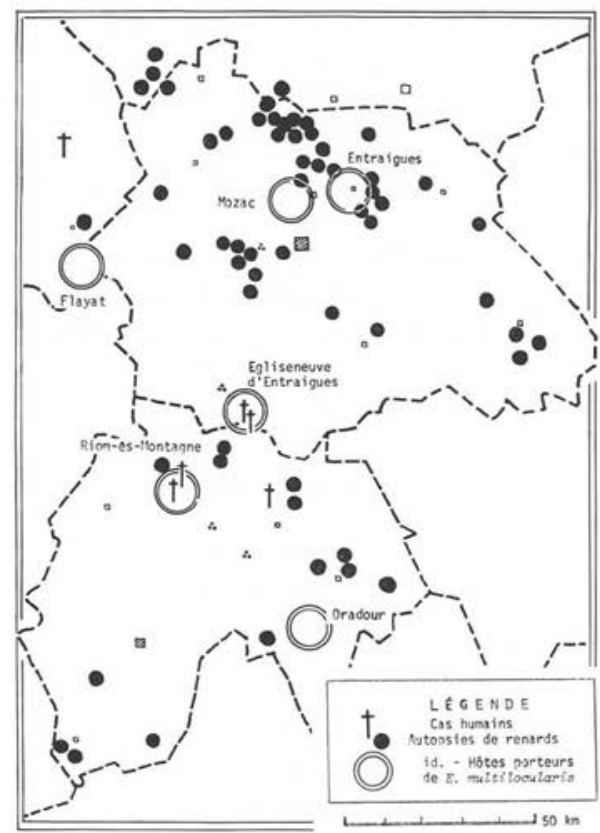

CARte no r. - Localisation en Auvergne des cas humains d'hydatidose alvéolaire hépatique, et des renards porteurs du tænia multiloculaire.

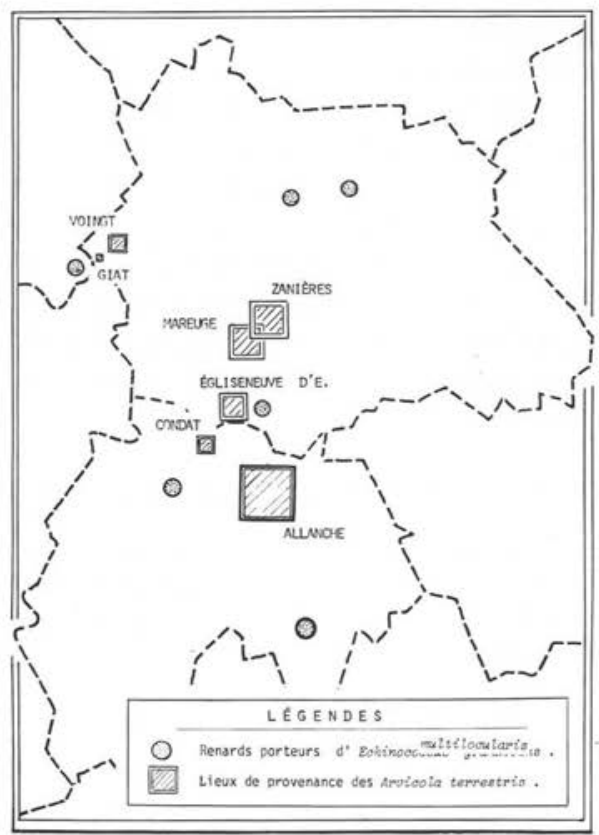

CARTE $\mathrm{n}^{\circ}$ 2. - Localisation en Auvergne des lieux de piégeage des Arvicola terrestris en I 98 I-I982.

selon les données du tableau IV et de la carte $n^{0} 2$. Les 23 grands campagnols parasités, constitués de 10 mâles et de 13 femelles, pèsent de 60 à $130 \mathrm{~g}$ selon le cas; pour la majorité d'entre eux (soit 19), il s'agit d'adultes de $90 \mathrm{~g}$ et plus.

TABLEAU IV. - Incidence géographique des hydatides alvéolaires observées chez les Arvicola d'Auvergne.

\begin{tabular}{|c|c|c|c|}
\hline Communes & $\begin{array}{l}\text { Incidence des } \\
\text { hydatides } \\
\text { alvéolaires }\end{array}$ & $\begin{array}{l}\text { Nombre de } \\
\text { captures }\end{array}$ & $\begin{array}{c}\% \\
\text { local des cas de } \\
\text { parasitisme }\end{array}$ \\
\hline Giat & 0 & 3 & - \\
\hline Voingt & 0 & 50 & - \\
\hline Zanières & 1 & 197 & $0,50 \%$ \\
\hline \multirow{2}{*}{\multicolumn{4}{|c|}{ Egliseneuve }} \\
\hline & & & \\
\hline d'Entraigues & 2 & 90 & $2,22 \%$ \\
\hline Allanche & $1 \overline{7}$ & 365 & $4,65 \%$ \\
\hline Condat & 2 & 51 & $3,92 \%$ \\
\hline Totaux & $\overline{23}$ & $\overline{943}$ & $\overline{2,44 \%}$ \\
\hline
\end{tabular}


L'incidence du parasitisme alvéolaire en fonction des dates de capture, tous lieux de piégeage confondus, figure dans le tableau $V$.

Les piégeages n'ont été conduits d'une façon suivie au cours de l'année que dans la commune d'Allanche. Les fluctuations du nombre des campagnols parasités, piégés dans une même prairie, sont considérables ainsi qu'en témoignent les données des tableaux VI et VII.

Sur les 23 cas d'hydatides alvéolaires recensés, deux sont fertiles, soit 8,69\% ; les deux campagnols correspondants ont été capturés en Août 1981 à Allanche et en Mars 1982 à Condat. Enfin, dans quatre cas, l'hydatide s'associe dans le foie de l'hôte à une larve mûre et vivante de Cysticercus fasciolaris.

TABLEAU V. - Incidence saisonnière du parasitisme alvéolaire des Arvicola en Auvergne.

\begin{tabular}{lccc}
\hline Dates & $\begin{array}{c}\text { Nombre } \\
\text { d'hydatides }\end{array}$ & $\begin{array}{c}\text { Nombre } \\
\text { d'autopsies }\end{array}$ & \% des prises \\
\hline Août 1981 & 1 & 123 & $0,81 \%$ \\
Oct. 1981 & 0 & 167 & $-\overline{7} \%$ \\
Janv. 1982 & 13 & 74 & $17,56 \%$ \\
Mars & 2 & 191 & $1,04 \%$ \\
Avril & 1 & 93 & $1,07 \%$ \\
Mai & 1 & 111 & $3,60 \%$ \\
Juil. & 1 & 33 & $3,03 \%$ \\
Août & 151 & $0,66 \%$ \\
\hline
\end{tabular}

TABLEAU VI. - Variations de la prévalence de l'hydatidose alvéolaire chez les Arvicola d'Allanche au cours de l'année.

\begin{tabular}{lrrrrr}
\hline \multirow{1}{*}{ Nombre } & \multicolumn{2}{c}{1981} & \multicolumn{2}{c}{1982} & \\
& Août & Octobre & Janvier & Mai & Total \\
\hline $\begin{array}{l}\text { des prises } \\
\text { des kystes }\end{array}$ & 37 & 167 & 74 & 87 & 365 \\
$\begin{array}{l}\text { lacéolaires } \\
\text { \% par rapport }\end{array}$ & 1 & 0 & 13 & 3 & 17 \\
\begin{tabular}{l} 
aux prises \\
\hline
\end{tabular} & 2,7 & 0 & 17,5 & 3,44 & 4,65 \\
\hline
\end{tabular}

\section{IV — Étude des pièces anatomiques}

\section{A - Aspects anatomo-pathologiques de l'organe parasité}

A l'autopsie des campagnols, dix des 23 lésions d'alvéolaire (soit 43,5\%) son reconnues comme telles d'après leur aspect, leur volume et leur couleur en surface du foie. Les autres, soient $13(56,5 \%)$ sont prélevées sous les appellations provisoires de " nodules kystiques » ou d' " abcès bactérien (?) ", du fait de leur très petite taille ou 


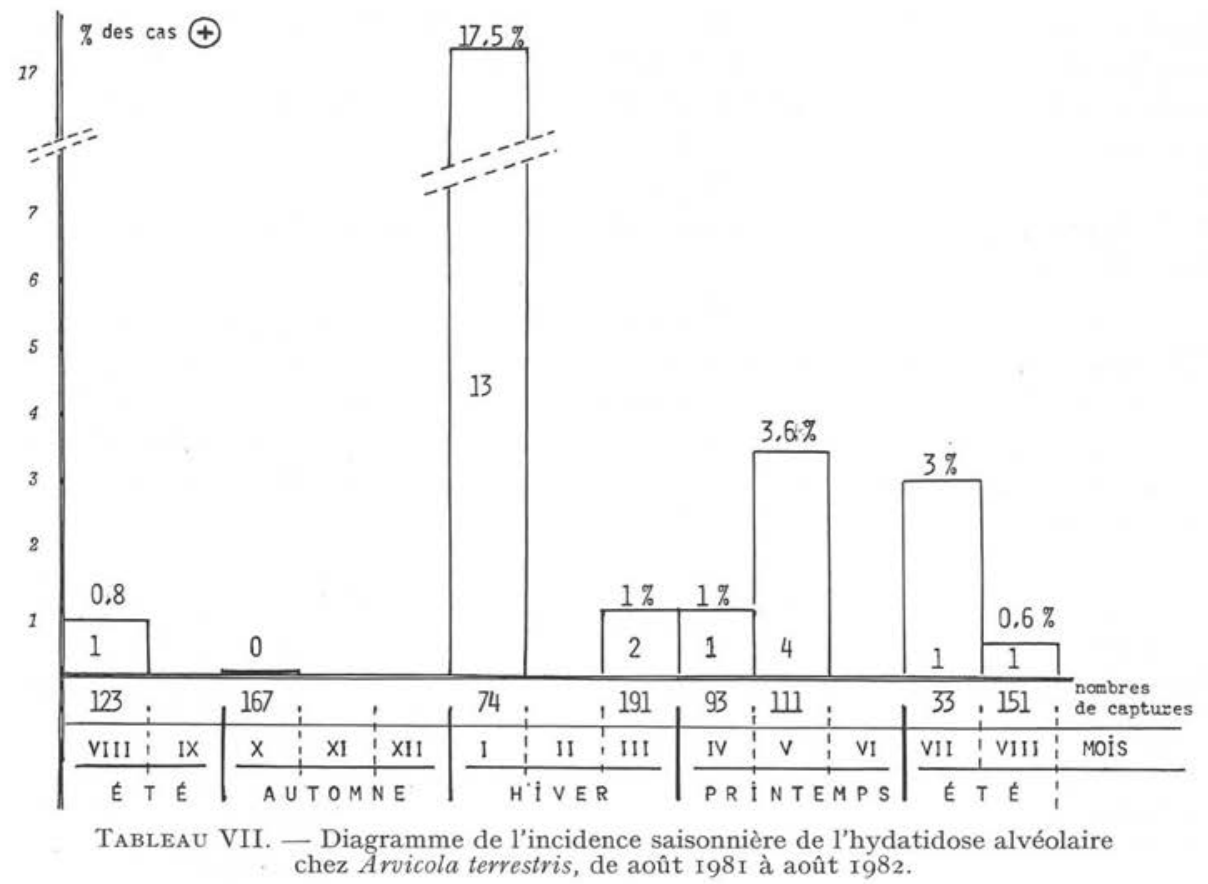

de leur aspect non caractéristique. Au moins une larve a été prélevée par hasard, avec un lobe porteur d'un cystique volumineux d'une autre espèce de cestode ; une autre évoquait par son aspect macroscopique la larve du Taenia tenuicollis Rud., 1819. Enfin, une lésion apparamment unique peut révéler plusieurs larves lors de la préparation du bloc pour l'histologie.

Le nombre exact des hydatides par hôte est difficile à préciser, que les lésions soient jeunes (du fait alors de leur discrétion) ou âgées (du fait alors d'un essaimage à distance toujours possible). Néanmoins deux foies nous sont apparus comme pouvant être parasités par deux larves indépendantes, et un par trois.

Les lésions parasitaires jeunes ou très jeunes ne modifient pas ou à peine la coloration naturelle du foie en surface ; elles surélèvent légèrement en dôme ou dépriment faiblement en cône ou en cratère la surface du lobe atteint, sur 0,5 ou $1,5 \mathrm{~mm}$ de diamètre. Ces altérations de surface ne permettent pas de soupçonner l'étiologie exacte de la lésion sous-jacente du parenchyme ; le diagnostic est porté à l'histologie. Les lésions âgées bien développées apparaissent sous plusieurs aspects. Le premier est celui d'une larve cysticerque de tænia de 5 à $15 \mathrm{~mm}$ de diamètre, circonscrite par une enveloppe résistante, opaque et blanc-jaunâtre, pouvant faire saillie de part et d'autre du lobe hépatique parasité. Le second aspect est celui d'une perle translucide, conséquence d'une paroi mince limitant une lésion cavitaire liquidienne sous-jacente. Le troisième aspect est celui de taches circulaires jaunâtres polycycliques, légèrement en 
relief, à l'image de taches de bougies. Les deuxième et troisième aspects des larves alvéolaires coexistent éventuellement dans un même foie. Des larves âgées essaiment enfin à proximité du foie, et se greffent sur des organes de la cavité abdominale (rate, péritoine), en affectant un aspect vésiculaire.

\section{B - Aspect histologique des larves d'Echinococcus multilocularis chez Arvicola terrestris}

Il a été procédé à un essai de classement des lésions hépatiques observées en histologie, en fonction de leurs tailles sur coupe et des morphologies générales. Cet essai figure dans le tableau VIII. Il permet d'évaluer la fréquence relative des divers aspects possibles des larves (inscrits sur le tableau sous la forme du numéro d'ordre de l'hôte d'origine). Les lésions hépatiques inférieures au millimètre de diamètre ont échappé aux investigations. TABleAU VIII. - Caractéristiques des hydatides alvéolaires des
Arvicola terrestris d'Auvergne.

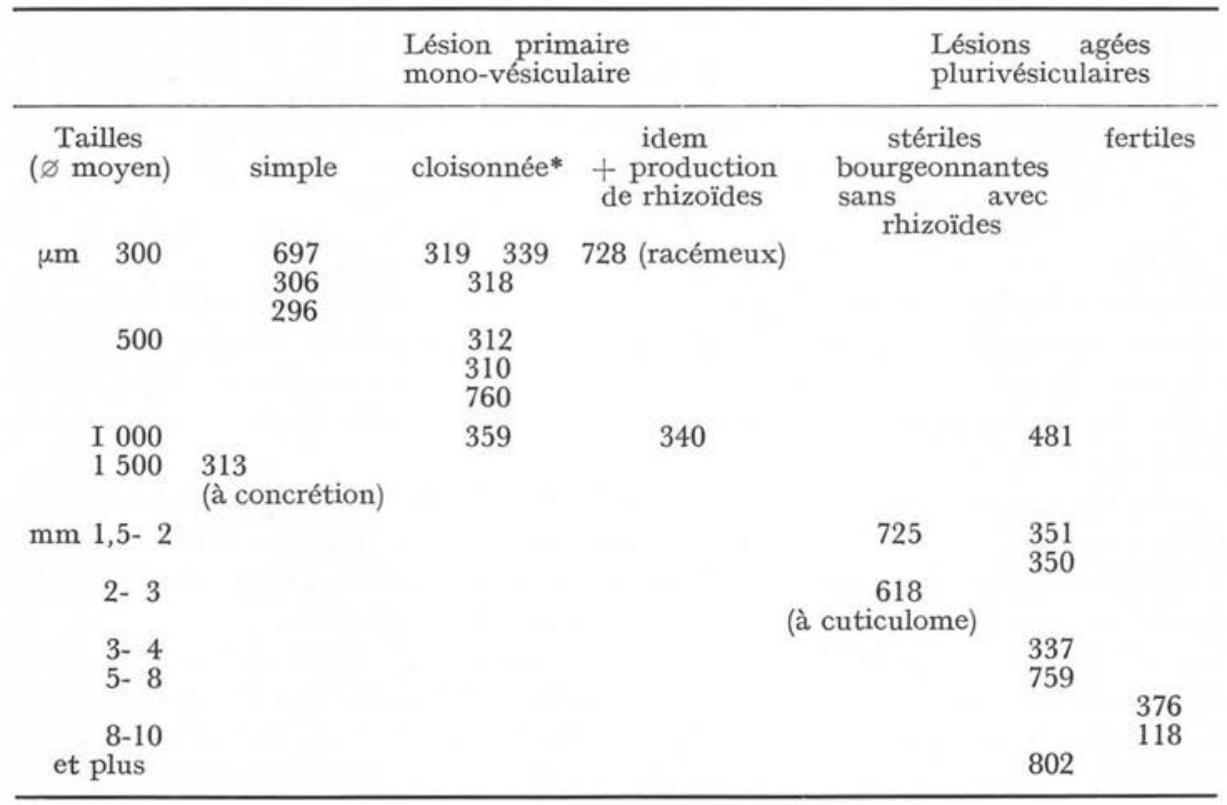

* " arabesques " de cuticule.

a) Cas des larves jeunes ( $P l . I$ ).

Les larves jeunes sont uniloculaires et se présentent comme des lacunes subcirculaires ou irrégulièrement ovoïdes du parenchyme hépatique. Les plus petites mesurent $100-200 \times 250-350 \mu \mathrm{m}$ de diamètre ; la plus grande simule un Cysticercus 
tenuicollis mesurant $1400 \times 1600 \mu \mathrm{m}$ de diamètre et contient une concrétion calcaire en grain de mil. Le type monovésiculaire pur est rare; il semble fugace; la larve montre en effet une tendance précoce : 1) à cloisonner le volume interne de sa cavité primaire par des festons ou des ponts de cuticule, qui dessinent dans la lumière des arabesques caractéristiques; 2) à bourgeonner des vésicules périphériques. Cette double tendance morphogénétique se poursuit au cours de la croissance du parasite et conduit à l'image histologique de l'hydatidose plurivésiculaire. Certaines larves jeunes associent enfin aux aspects décrits ci-dessus un troisième type de lésion, déjà perceptible sur les tranches de matériel fixé examinées au grossissement des loupes binoculaires (XI 0 à 20), celui de la lésion hépatique " en tranche de pain d'épice "; il est consécutif à la présence et au développement des multiples stolons ou rhizoïdes du parasite au sein d'un vaste tissu réactionnel de l'hôte. Il conduit au troisième type histologique possible trouvé chez les Arvicola, le type alvéolaire.

Dans tous les cas, le diamètre apparent de la lésion hydatique des trois types ci-dessus est considérablement augmenté par la réaction tissulaire de contact qui est constante, précoce et marquée. Celle-ci double les dimensions des parasites jeunes dans de nombreux cas, les triplant ou les quadruplant dans quelques autres. Ainsi une

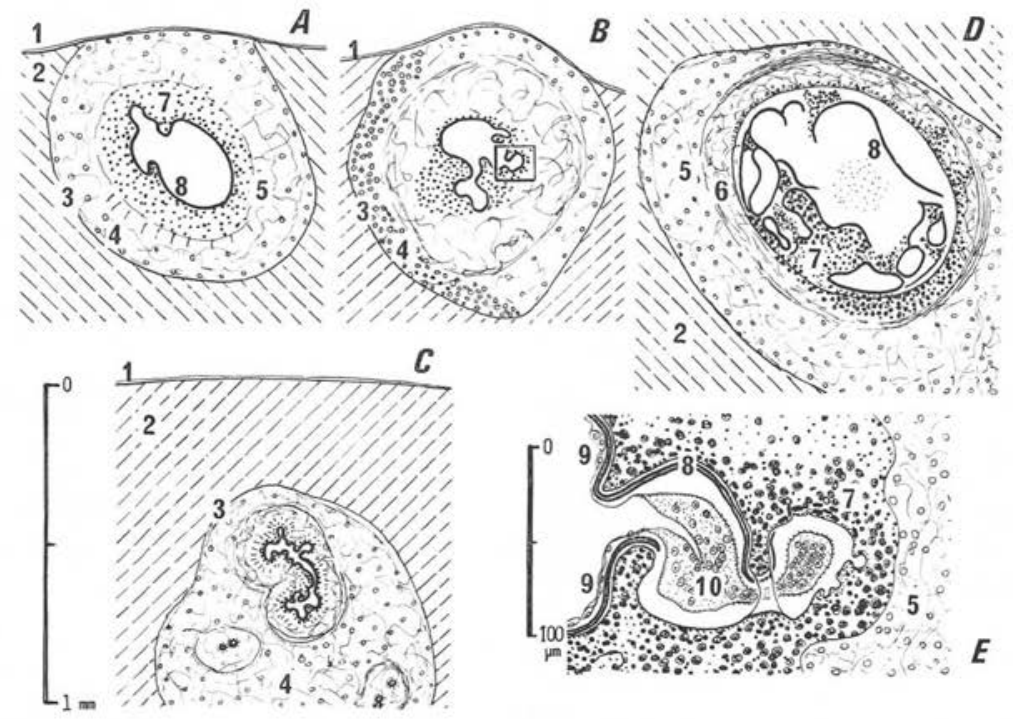

Planche I. - Aspects semi-schématiques des hydatides alvéolaires du foie d'A rvicola terrestris en Auvergne.

Légendes communes aux figures. $I$ : capsule de Glisson. 2 : foie sain. 3 : limite externe de l'adventice. 4 : réaction histio-lympho-monocytaire, $s:$ réaction fibro-conjonctive lâche. $6:$ réaction fibro-conjonctive dense. 7 : tissu granuleux nécrosé. 8 : cuticule du parasite. $9:$ membrane proligère. 10 : tissu plasmodial.

I) larves jeunes. A : larve uniloculaire simple. B et C : larves uniloculaires racémeuses. D : larve uniloculaire cloisonnée par un développement de la cuticule en arabesques multiples. E : détail (portion encadrée de la fig. B). Progression du plasmode parasitaire dans le tissu réactionnel de l'hôte. 
hydatide monovésiculaire de $200 \times 250 \mu \mathrm{m}$ de diamètre conduit à une lésion hépatique de $400 \times 500 \mu \mathrm{m}$ environ; une autre de $800 \times 1200 \mu \mathrm{m}$, à une lésion de $2,5 \times 3,5 \mathrm{~mm}$ de diamètre. Le tableau VIII met en évidence que les types plurivésiculaires et alvéolaires prédominent dans la série de 23 hôtes parasités capturés en Auvergne et, en particulier, lorsqu'il s'agit de larves âgées de grande taille.

b) Cas des larves âgées ( $P l . I I)$.

Les larves âgées sont fertiles ou non.

1) les deux larves âgées fertiles sont apparues, en Auvergne de deux types différents.

Le premier type semble résulter de la continuation du processus de développement anarchique de la larve dans le tissu réactionnel de l'hôte, s'accompagnant de la formation de vésicules multiples dont les plus nombreuses sont stériles, et dont quelques-unes sont envahies de la prolifération abondante de la membrane cuticulaire (" cuticulomes" du tableau VI). Les rares protoscolex présents en quelques points localisés de certaines hydatides se situent au sein de vésicules plus ou moins emboitées les unes dans les autres, à la façon des poupées russes. La membrane proligère est du type plat. Quelques vésicules ne comportent plus que des scolex dégénérés.

Le second type résulte d'un développement assagi de la larve qui conduit à la naissance d'un nombre réduit de vésicules régulièrement ovoïdes, de grande taille relative $(3,1 \times 1,5 \mathrm{~mm} ; 7,5 \times 5,5 \mathrm{~mm} ; 13 \times 8,5 \mathrm{~mm})$ que réunit un étroit pertuis, et bien encapsulées. L'ensemble offre l'aspect d'un kyste de Cysticercus fasciolaris, ou mieux d'une hydatide pluriloculaire de E. granulosus. Les cavités sont liquidiennes ; la membrane proligère est haute et se creuse de centaines de vésicules proligères pourvues de milliers de protoscolex bien vivants. Ces cystiques, du fait de leur isolement parfait, paraissent bien tolérés par le tissu hépatique, à l'égal d'un $C$. fasciolaris par exemple, d'ailleurs présent dans un autre lobe du même foie et entouré d'une adventice exactement de même type.

2) Les larves âgées acéphalocystes s'apparentent au premier type décrit ci-dessus.

\section{Histologie des lésions hépatiques}

L'examen histologique pose le diagnostic de la nature alvéolaire des lésions du foie des Arvicola. La succession dans le sens centripète des lésions observables est la même chez tous les hôtes.

A) L'adventice. En périphérie se situent les travées d'hépatocytes sains, souvent légèrement comprimés au voisinage des réactions tissulaires engendrées par la présence de la larve. Puis s'observe une réaction conjonctive fibreuse lâche et inflammatoire pas ou peu vascularisée. Dans ses mailles affluent des leucocytes, parfois en nombre extrêmement élevés au moins en certains points de la périphérie. A cette réaction inflammatoire succède une réaction fibroconjonctive un peu plus dense, à direction générale concentrique à la larve ou en arceaux enchevêtrés ; elle est parfois riche en cellules géantes; par contre, la réaction inflammatoire y est nettement atténuée. A proximité du parasite s'observe souvent une réaction de dégénérescence conjonctive et de nécrose préparant la calcification. Elle se traduit par l'apparition 

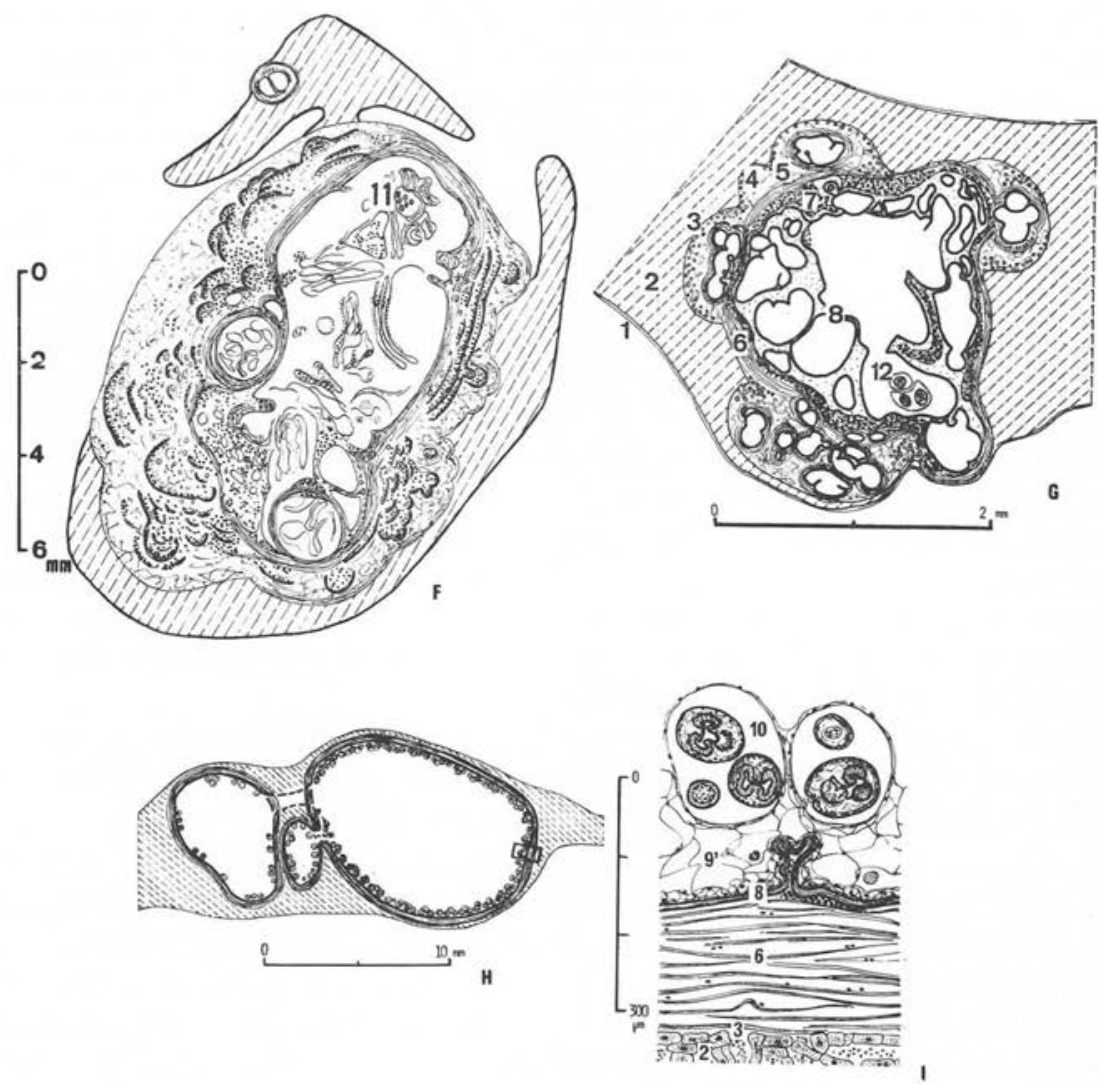

Planche II. - Aspects semi-schématiques des hydatides alvéolaires du foie d'A rvicola terrestris en Auvergne.

2) Larves âgées multiloculaires (Condat, 1982). F : Envahissement de la cavité primaire par une profusion de membranes cuticulaires ("cuticulome»). - I I : amas de scolex dégénérés ; vésicules proligères disparues. G : id. - I2 : deux vésicules proligères fertiles vivantes.

3) Larves pauci-vésiculaires (Allanche, 198I). H : tranche de foie parasité. Larve constituée de trois vésicules bien circonscrites interconnectées. Nombreuses vésicules proligères fertiles. - I : id. Détail (portion encadrée de $\mathrm{H}$ ). $-9^{\prime}$ : parenchyme proligère. - Io : vésicules proligères parenchymateuses non pédonculées et protoscolex.

d'un matériel granuleux bien colorable se densifiant en direction centripète ; ce dernier peut soulever localement la membrane cuticulaire et se laisser emprisonner dans ses festons.

La réaction de l'hôte n'est jamais spécifique d'une hydatide alvéolaire.

B) Le parasite. Les tissus limitants du parasite sont représentés par la membrane cuticulaire anhiste, à aspect de membrane pellucide mince uni-ou pluri-stratifiée. Celle-ci dessine souvent de larges festons se décollant de la périphérie, pour pénétrer profondément dans la cavité kystique et se collaber à la paroi d'en face ; cette cuticule 
peut également se délaminer pour établir à distance de multiple anastomoses avec elle-même. Ainsi se constitue sur les coupes un cloisonnement multiple mais souvent partiel de la cavité kystique considérée. La membrane proligère est figurée par un filament nucléé de place en place, étroitement appliqué contre la membrane cuticulaire ; cette membrane n'apparaît pas présente au niveau de toutes les surfaces libres (par disparition spontanée, ou à la suite d'un artéfact technique possible) ; elle s'épaissit en réseau localement, dans les creux des festons cuticulaires étrécis. Elle engendre parfois des corpuscules calcaires. En périphérie de certaines alvéoles, et au contact direct des tissus de l'hôte, en certains points privilégiés, la membrane proligère se résout en un plasmode aux noyaux nucléolés volumineux; en arrière du front de progression de cette masse plasmodiale, l'élaboration d'une mince couche de cuticule tapisse la cavité néo-formée. Les stolons ou rhizoïdes du parasite procèdent du même processus de genèse ; l'aspect histologique est comparable.

La détermination de nos larves acéphalocystes jeunes s'est basée sur l'observation des structures morphologiques définies ci-dessus, comparées à celles des hydatides alvéolaires âgées typiques et à celles, très différentes, des cystiques hépatiques de quatre espèces de tænia, également parasites des foies de campagnol, et évoqué dans un autre article (Deblock et Pétavy, 1983).

Hormis les formations membranaires décrites, la lumière des alvéoles apparaît optiquement vide, sauf dans les cas où figure un fin dépôt de matière fibrinoïde, traduction histologique de la substance gélatineuse observable in vivo.

C) Les vésicules proligères. Dans les deux larves fertiles récoltées s'observent deux types différents de formation des vésicules proligères. Dans l'une, la membrane proligère demeure basse et fournit dans la cavité alvéolaire une vésicule proligère pédiculisée et isolée, fertile de 3 à 4 proto-scolex visibles simultanément sur la coupe histologique. Dans l'autre type, la membrane proligère se développe en un parenchyme proligère relativement haut (100 à $220 \mu \mathrm{m}$ ) dont les mailles s'élargissent pour donner naissance laisser croître et abriter un ou plusieurs proto-scolex. La vésicule apparaît ainsi, à la suite du processus décrit de son élaboration, à demi-enchâssée dans la couche épaisse de parenchyme proligère ${ }^{2}$.

D) Le diagnostic histologique de la nature de larve non fertile ne pose pas de difficulté particulière lorsqu'elle engendre les stolons ou rhizoïdes caractéristiques de l'espèce, en périphérie des lésions vésiculaires initiales. Une réaction fibro-conjonctive considérable du tissu parasité accompagne toujours ces formations chez les Arvicola.

\section{V - Résumé et conclusion}

Houin et coll., 1982 capturent dans le Jura français (région de la Franche-Comté) au cours de l'année 1980, 1.853 Arvicola terrestris dont 5, soit 0,42 ; sont porteurs d'hydatides alvéolaires hépatiques. 152 autres rongeurs en provenance de Savoie sont

2. Tailles des vésicules proligères : de IOO-I30 à $200-250 \mathrm{~m}$ de $\varnothing$. Protoscolex : I IO-I30 $80 \mu \mathrm{m}$ de $\varnothing$. Rostre pourvu de deux couronnes de io à $\mathrm{I} 3$ crochets ( $=20$ à 26 crochets) ; grands : 25-29 $\mu \mathrm{m}$; petits : $20-24 \mu \mathrm{m}$. 
indemnes de la parasitose. Notre enquête menée dans le Massif Central (région de l'Auvergne) d'août 1981 à août 1982, confirme qu'en France, à la différence de l'Europe Centrale, c'est bien ce rongeur qui peut être incriminé jusqu'à présent dans le cycle naturel du parasite Echinococcus multilocularis. L'examen parasitologique révèle en effet que 23 d'entre eux sur 943 (soit 2,44\%) hébergent une ou plusieurs larves d'hydatides multiloculaires hépatiques. 8,7\% des larves sont fertiles (2 sur 23) et $60 \%$ (soit 14 sur 23 ) mesurent moins de $1.500 \mu \mathrm{m}$ de diamètre. Ces 23 larves sont étudiées du point de vue anatomo-pathologique et histologique L'observation de l'aspect de la paroi kystique sur coupe se révèle le seul moyen efficace de déterminer la nature alvéolaire des larves encore jeunes et uniloculaires, essentiellement par la structure lamelleuse de la membrane cuticulaire et par les arabesques qu'elle décrit dans la cavité primaire des lésions.

Selon les communes, le pourcentage des rongeurs infestés varié de zéro (Voingt) à 0,5 (Zanières, Mareuge), 2,2\% (Égliseneuve d'Entraigues), 3,92\% (Condat) et $4,65 \%$ (Allanche). L'incidence du parasitisme apparaît donc, dans les limites de l'enquête, comme nulle, faible, ou assez élevée selon les communes. Les foyers les plus favorables à la parasitose animale restent actifs toute l'année, ni l'été ni l'hiver ne semblant faire obstacle au déroulement du cycle évolutif du parasite. L'endémie parasitaire paraît se maintenir, dans les biotopes actifs, aux environs de 1 à $2 \%$ de la population des Arvicola (extrêmes : 0,5 à 4,6\%). Cette incidence traduit une dissémination renouvelée et permanente dans la nature, sur de vastes surfaces, des embryophores infestants du tænia échinocoque multiloculaire à partir de l'hôte définitif. Sur cette endémie diffuse et stable vient parfois se greffer une épidémie, comme à Allanche (en janvier 1982, tableau VI : $17 \%$ de cas), sans doute consécutive aux visites renouvelées d'un ou de plusieurs renards fortement parasités et attirés par la densité élevée des rongeurs dans la prairie prospectée.

L'efficacité de la transmission du parasite semble toutefois compromise par le grand nombre de larves stériles : $90 \%$ sont acéphalocystes le jour de la capture de l'hôte. Le renard doit donc ingérer 10 Arvicola parasités pour avoir une chance de contracter un téniasis, soit 500 campagnols $^{3}$. Une raison de cette stérilité réside sans doute dans le fait que beaucoup de larves sont trop jeunes, donc trop réduites en volume pour donner naissance à des proto-scolex ; mais les larves âgées plus volumineuses ne deviennent pas nécessairement fertiles non plus. Faut-il incriminer une mauvaise adaptation hôte-parasite en dépit d'une excellente réceptivité initiale des rongeurs? Les piégeages de la prairie d'Allanche le feraient croire (tableau VI). L'épidémie de janvier 1982, avec $17,5 \%$ de cas sur 74 campagnols, est suivie en mai de la mise en évidence d'un pourcentage de cas cinq fois moins élevé $(3,4 \%$ de cas sur 87 campagnols), et de l'absence de larve devenue fertile malgré les cinq mois séparant les deux piégeages. Il faudrait donc admettre ou bien une maturation extrê-

3. Cette quantité de campagnols représentant une masse de $50 \mathrm{~kg}$ de viande est ingérable en un mois environ. Dans les zones de prolifération des rongeurs, les services de protection des végétaux du Puy-de-Dôme estiment la densité de population à 300 ou 400 animaux par hectare de prairie; quant à la densité des renards en Auvergne, on l'évalue entre quinze et vingt animaux pour rooo ha. 
mement lente du parasite, ou bien une guérison spontanée de la plupart des lésions débutantes de janvier, suivie de l'infestation plus récente des animaux piégés en mai. Dans les deux hypothèses, l'hôte n'apparaît guère favorable au parasite.

Quelle que soit la réalité des faits, la probabilité élevée de l'infestation des Arvicola augmente les chances du parasite de rencontrer un individu de réceptivité convenable, médiocre ou excellente. Dans le cas d'une réceptivité médiocre, la larve fournit çà et là, dans quelques vésicules, de rares protoscolex à l'origine d'une infestation discrète du renard prédateur (4 renards sur 5 selon Pétavy et coll., 1980) ; dans le cas d'une réceptivité excellente, la larve devient prolifique et engendre des milliers de protoscolex à l'origine d'une infestation massive du prédateur (1 cas en 1980 ibid., et 1 cas en 1982 : cf. tableau $I I$ ). Celle-ci entraînera en quelques semaines un accroissement considérable du stock des œufs du parasite mis à la disposition des rongeurs dans la nature, éventuellement source d'une explosion épidémique locale de l'enzootie. L'hydatide envahissante, pathogène pour l'hôte et peu fertile, pourra handicaper le rongeur parasité et faciliter sa prédation, ou accélérer sa mort spontanée. L'hydatide assagie, bien tolérée et prolifique, ne devrait ni écourter la longévité ni amoindrir la vitalité de son hôte. La conservation du virus dans la nature pourrait peut-être, en Auvergne du moins, s'expliquer par le jeu simultané de ces deux types de comportement des hôtes intermédiaires vis-à-vis du parasite, en dépit de la longévité relativement courte du tænia adulte chez le renard (estimée de 3 à 6 mois).

Les risques de contamination humaine apparaissent extrêmement faibles si l'on s'en tient aux statistiques établies à partir des cas chirurgicaux (six cas en Auvergne depuis 1977) et qu'on compare ce nombre restreint de malades à l'exubérance biologique du parasite mise en évidence.

La perplexité du parasitologiste s'accroît cependant lorsqu'il capture deux Arvicola porteurs chacuns d'une hydatide hépatique - dont une fertile - sous les pelouses d'un terrain de camping d'un niveau de confort très honorable et très fréquenté par les touristes et les familles de vacanciers. Son inquiétude s'amplifie quand il considère que les Arvicola constituent des populations sédentaires, que les rongeurs se sont donc contaminés nécessairement sur place, et que les pièges ont été disposés au milieu de terriers situés sous l'emplacement même des tentes montées lors des dernières vacances... Si les risques de contamination sont faibles, ils ne sont certes pas nuls. Sur une surface inférieure à cinq hectares de la commune d'Égliseneuve d'Entraigues, deux cas humains d'alvéolaire hépatique, l'autopsie d'un renard porteur de nombreux cestodes adultes ovifères, et la capture de deux Arvicola hébergeant l'un et l'autre une larve hépatique alvéolaire, illustrent parfaitement semble-t-il, le dynamisme du foyer de la parasitose et le danger potentiel de contamination encouru par la population du Massif Central ou par celle qui vient s'y détendre à la belle saison.

Remerciements. Ce travail a reçu le support financier de la D.G.R.S.T. Messieurs Pradier et Vallat du service de Protection des Végétaux du Ministère de l'agriculture (section du Puy-de-Dôme) et Messieurs L. C. Vignon et G. Papon doivent être remerciés de leur aide efficace apportée par leur connaissance des aires de prolifération des rongeurs et pour leur habileté sur le terrain lors des piégeages. 


\section{BIBLIOGRAPHIE}

Bourée P., Seguin M., Labayle D., Longchal C. - Échinococcose alvéolaire multiple ; infestation probable dans la Creuse. Nouv. Presse Med., I978, 7, 2480.

Deblock S., PÉTAVy A. F. - Les larves hépatiques de cestode parasites du grand campagnol Arvicola terrestris en Auvergne (France). 1983, (à paraittre).

HouIN R., DENIAU M., LIANCE M. - Arvicola terrestris (L.) premier rongeur trouvé naturellement infesté par Echinococcus multilocularis Leuckart, 1863 en France. C.R. Acad. SG. Paris, D, 1980, 290, I 269-1 271 .

Houin R., Deniau M., Liance M., Puel F. - Arvicola terrestris an intermediate host of Echinococcus multilocularis in France; epidemiological consequences. Intern. J. Parasitol, I982, $12,593-600$.

PÉtAVy A. F., DEBLock S. - Helminthes du renard commun (Vulpes vulpes L.) dans la région du Massif Central (France). Ann. Parasitol. Hum. Comp., I980, 55, 379-391.

Rey M., Morin B, PÉtAyy A. F., CAMbon M., BarIL A. - Premières observations auvergnates d'échinococcose alvéolaire. Nouv. Presse Med., 1977, 6, 4070-4072,

Rey M., Lusson J. R., Baril A., Pétavy A. F. et coll. - Présence en Auvergne de l'échinococcose alvéolaire. Rev. Med, Clermont-Ferrand, 1978, s, 44I-45I. 